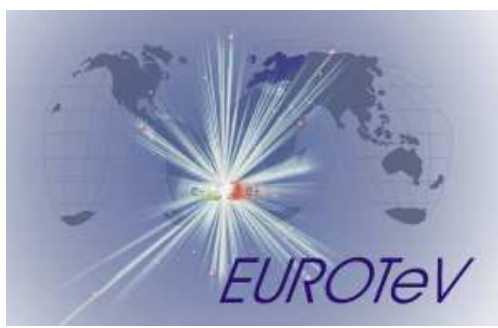

\title{
A PROTOTYPE ENERGY SPECTROMETER FOR THE ILC AT END STATION A IN SLAC
}

\author{
A. Lyapin, F. Gournaris, B. Maiheu, D. Miller, M. Wing, \\ University College London, UK
}

M. Chistiakova, Yu. Kolomensky, M. Sadre-Bazzaz, E. Petigura, University of California in Berkeley and LBNL, USA

M. Slater, M. Thomson, D. Ward, University of Cambridge, UK

H.-J. Schreiber, M. Viti, DESY, Germany

S. Kostromin, N. Morozov, V. Duginov, JINR, Dubna, Russia

M. Hildreth, University of Notre-Dame, USA

S. Boogert, G. Boorman, Royal Holloway University of London, Egham, UK

C. Adolphsen, R. Arnold, C. Hast, D. McCormick, Z. Szalata, M. Woods, SLAC, USA

June 20, 2007

\begin{abstract}
The main physics program of the International Linear Collider requires a measure ment of the beam energy with a relative precision of the order $10^{-4}$ or bett er. A magnetic spectrometer using high resolution beam position monitors (BPMs) has been proposed to achieve this goal. A prototype spectrometer chicane employi ng four dipole magnets is currently under development at the End Station A in SL $\mathrm{AC}$, intending to demonstrate the required resolution and stability of this metho $\mathrm{d}$ and investigate possible systematic effects and operational issues. This contr ibution reports on the successful commissioning of the beam position monitor sys tem and the resolution and stability achieved. Also, the initial results from a run with a full spectrometer chicane are presented.
\end{abstract}

*al@hep.ucl.ac.uk 


\title{
A PROTOTYPE ENERGY SPECTROMETER FOR THE ILC AT END STATION A IN SLAC*
}

\author{
A. Lyapin ${ }^{\dagger}$, F. Gournaris, B. Maiheu, D. Miller, M. Wing, University College London, UK \\ M. Chistiakova, Yu. Kolomensky, M. Sadre-Bazzaz, E. Petigura, \\ Berkeley and Lawrence Berkeley National Laboratory, USA \\ M. Slater, M. Thomson, D. Ward, University of Cambridge, UK \\ H.-J. Schreiber, M. Viti, DESY, Germany \\ S. Kostromin, N. Morozov, V. Duginov, JINR, Dubna, Russia \\ M. Hildreth, University of Notre-Dame, USA \\ S. Boogert, G. Boorman, Royal Holloway University of London, Egham, UK \\ C. Adolphsen, R. Arnold, C. Hast, D. McCormick, Z. Szalata, M. Woods, SLAC, USA
}

\section{Abstract}

The main physics program of the International Linear Collider requires a measurement of the beam energy with a relative precision of the order $10^{-4}$ or better. A magnetic spectrometer using high resolution beam position monitors (BPMs) has been proposed to achieve this goal. A prototype spectrometer chicane employing four dipole magnets is currently under development at the End Station A in SLAC, intending to demonstrate the required resolution and stability of this method and investigate possible systematic effects and operational issues. This contribution reports on the successful commissioning of the beam position monitor system and the resolution and stability achieved. Also, the initial results from a run with a full spectrometer chicane are presented.

\section{INTRODUCTION}

The International Linear Collider (ILC) electronpositron collider is designed to perform precision particle physics measurements up to $500 \mathrm{GeV}$. A beam energy spectrometer is one of the key diagnostic devices for measuring the beam energy on a bunch-by-bunch and bunch-train basis.

The energy spectrometer proposed for the ILC is based on the system successfully used at LEP, where an accuracy of $1.7 \times 10^{-4}$ was achieved [1]. The LEP and ILC spectrometer monitor the beam deflection produced by a dipole magnet. The baseline ILC spectrometer design uses two magnets to produce a beam displacement, while two more dipoles return the beam to the nominal accelerator orbit. In the case of four identical magnets, the beam energy is then given by

$$
E=\frac{e c L \int B \mathrm{~d} l}{x},
$$

where $L$ is the distance between the magnets, $x$ the beam

\footnotetext{
* This work was supported by the Commission of the European Communities under the 6th Framework Programme "Structuring the European Research Area", contract number RIDS-011899

† al@hep.ucl.ac.uk
}

displacement, $\int B d l$ the integral magnetic field in each magnet.

The ILC spectrometer design has additional requirements on the maximum bending angle, to avoid emittance growth. This results in more demanding requirements on the BPMs compared to LEP. Thus, simple stripline or button BPMs have to be replaced with higher performance cavity BPMs.

It is known that beam tilts produce signals in the BPMs that be mistaken for the beam displacement, and thus introduce systematic errors in energy measurement. For this reason, a four-magnet chicane system, which maintains the beam axially with respect to the axes of all cavities, is preferable over a more conventional (as at LEP) threemagnet design. Additionally, in a four-magnet chicane the field can be varied from -Bmax to +Bmax to investigate systematic effects, without changing beam orientation.

A test beam experiment T-474 has been setup at the Stanford Linear Accelerator Center (SLAC) in End Station A beamline to demonstrate the performance of the spectrometer with a $28.5 \mathrm{GeV}$ beam. Our focus is on studies of the stability of various components of the system and relative measurements of the energy, in order to identify possible spectrometer configurations and operation scenarios at the ILC. The first results are reported in this contribution.

\section{TEST SETUP}

Two values need to be measured in order to determine the beam energy in the chicane: $x$, the offset of the beam from its initial orbit, and $B d l$, the integral magnetic field.

In order to study the precision of orbit reconstruction and resolution limits of the cavity BPMs, 8 BPMs were installed in ESA beamline, shown in Fig. 1. In 2007 four dipole magnets bending the beam horizontally (X direction) completed the setup to a full model of the proposed spectrometer with a relocation of the BPMs (Fig. 2).

Three types of S-band cavity BPMs were installed, 3 prototype BPMs for the ILC main linac (BPMs 3, 4 and 5) and 5 SLAC A-line and linac type BPMs [2] (BPMs $1,2,9,10$ and 11). The ILC prototype is a cylindrical cavity with a waveguide coupling system providing signals 


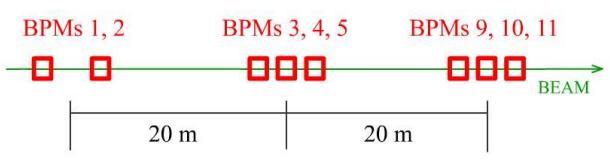

Figure 1: Setup of the T-474 experiment as in 2006, squares represent BPMs and triangles dipoles. (not to scale and coordinates approximate)

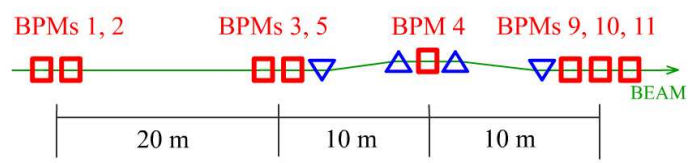

Figure 2: Setup of the T-474 experiment as in 2007.

for both $\mathrm{X}$ and $\mathrm{Y}$ and supressing unwanted modes. SLAC BPMs consist of 2 rectangular cavities, one for $X$ and one for $Y$ direction, and a cylindrical cavity providing the phase and the charge information.

BPM 4 was instrumented with an X-Y mover system allowing for precise calibration of this BPM and crosscalibration of the rest of the BPMs in the setup. The mover system was also used for tracking the beam while the chicane magnets were operated. A high precision interferometer system monitored the horizontal motion of BPMs 3, 4 and 5.

The BPM signal processing consisted of RF electronics mixing $2.9 \mathrm{GHz}$ BPM signals down to about $80 \mathrm{MHz}$. The downmixed signals were digitized at $119 \mathrm{MHz}$ (synchronous to the main linac RF system) using 14 bit ADCs. Further processing was performed offline to extract the position information from the recorded waveforms.

The SLAC linac provided bunches of up to $2 \cdot 10^{10}$ electrons, at a repetition frequency of $10 \mathrm{~Hz}$, parasitically to PEP-II injection. The transverse RMS beam size through the spectrometer was of order $200 \mu \mathrm{m}$ vertical and $500 \mu \mathrm{m}$ horizontal; the RMS bunch length is $500 \mu \mathrm{m}$. The beam orbit can be stabilised with a BPM-corrector feedback system. Feedback correctors and later also additional Helmholtz coils could be used to move the beam by a known amount and calibrate the BPMs.

\section{PERFORMANCE OF THE BPM SYSTEM}

We commissioned the BPM system in three runs in 2006, in which we investigated the resolution of individual BPMs, precision of the orbit determination and long term precision of the beam position measurement. The resolution of the BPMs was measured by comparing the position measured by the BPM under study and the measurements from the other BPMs in the system.

The resolution of the BPM is the minimum change of the beam position the BPM can detect in a single pulse. Data were collected for a relatively large number of ma- chine pulses obtained in a short period of time to reduce the effect of instabilities and drifts. The results are listed in Table 1. Sub-micron resolutions were measured for most of the BPMs apart from BPMs 1 and 2. Whose signals had to be carried over a longer distance cable than for the rest of the electronics in the setup, which resulted in a higher attenuation. Also BPM 4 which was mounted on a mover and suffered from a larger mechanical vibration.

We also defined a "linked resolution", which is the precision of the orbit fit from BPMs 1, 2, 9, 10, 11 extrapolated to the location of BPM 4 and compared with BPM 4 position measurement. This linked resolution was found to be less than $1 \mu \mathrm{m}$, meeting the requirements for successful spectrometer tests.

Table 1: BPM resolutions measured in 2006 running.

\begin{tabular}{|l|c|c|}
\hline BPM & Resolution in $\mathbf{X} \mu \mathbf{m}$ & Resolution in Y, $\mu \mathbf{m}$ \\
\hline 1,2 & 1.64 & 4.71 \\
3 & 0.49 & 0.50 \\
4 & 1.26 & 1.12 \\
5 & 0.59 & 0.44 \\
9 & 0.28 & 0.34 \\
10 & 0.16 & 0.20 \\
11 & 0.28 & 0.25 \\
Linked & 0.43 & 0.37 \\
\hline
\end{tabular}

Ideally, the accuracy of the position measurement would be the same as the resolution of the BPM. However on large time scale environmental effects such as temperature changes cause the BPM system to exhibit position drifts. Temperature changes can effect the gain and phase response of electronics, as well as directly effect the mechanical stability of the BPM mounting and support. As a result the position measurement and hence the orbit reconstruction become less precise. This has a crucial impact on the operation of the energy spectrometer as the precision of the energy measurement would stay within the desired value for only a limited time before a new calibration of the BPM system is required.

The results of one hour long measurement providing an idea of the scale of the longer term drifts is shown in Fig. 3. Here the same measurement as for the linked resolution has been carried out a number of times. The RMS of all measurements shown in the top plot does not change much (as expected), but the residual (bottom plot) does vary, indicating drifts. As can be seen, the drifts over 1 hour were within $\pm 1 \mu \mathrm{m}$ which is sufficient for the initial spectrometer tests, but must be improved in the future to achieve the required accuracy for the energy measurement.

A calibration system periodically sending a burst of RF oscillations to the electronics on an unfilled machine pulse, is currently being commissioned at ESA. Changes in gain and phase shift of the RF electronics, correlated with temperature variations, were clearly visible in the data taken in March 2007. Correcting for these drifts should improve the accuracy of the BPM data. 

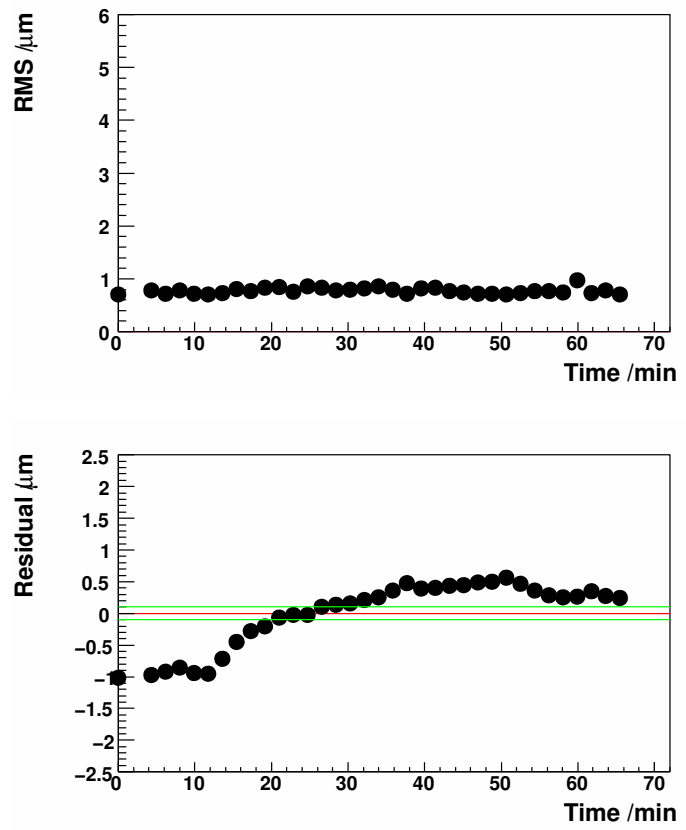

Figure 3: Precision of the BPM system over a period of 1 hour

\section{CHICANE MAGNETS}

In 2007, we installed and commissioned four dipole magnets in the ESA beamline. Measurements at the SLAC magnetic measurement facility, found the field integral to be uniform to the level of $10^{-4}$ in a region of $\pm 15 \mathrm{~mm}$ horizontally, with the stability of the integral about $100 \mathrm{ppm}$. The field integral was found to change by 60 ppm per degree $\mathrm{C}$, which is acceptable for these tests. The nominal current for a $5 \mathrm{~mm}$ beam offset is $150 \mathrm{~A}$, which corresponds to an integrated field of $0.117 \mathrm{~T} \cdot \mathrm{m}$. The magnets are instrumented in ESA with Hall and NMR probes to monitor the magnetic field. In addition we monitor the stray magnetic fields outside the magnets with low-field flux-gate magnetometers. A part of the ESA beamline with one of the magnets is shown in Fig. 4. A more detailed insight into magnet measurements can be found in [3].

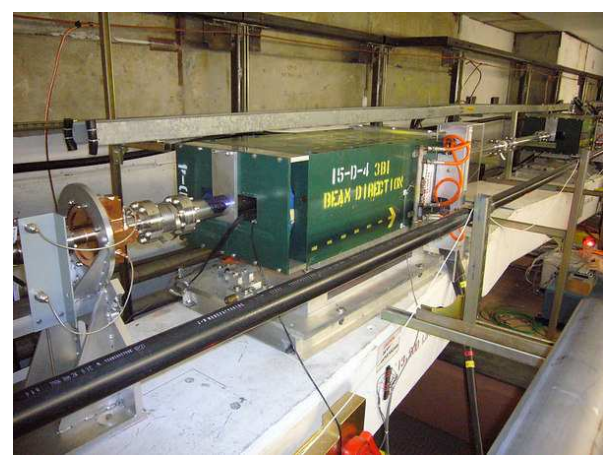

Figure 4: The first chicane magnet and BPM5 in the ESA beamline.

\section{FIRST ENERGY MEASUREMENTS}

In March 2007 we took data with the full spectrometer chicane. The BPM data was used to predict the position of the beam at the location of BPM 4 in the middle of the chicane. This was compared to the actual reading of BPM 4 to find the beam deflection, together with the data from the magnetic probes, resulting in first energy measurement.

The SLAC linac control system allows the setpoints of the energy feedback to change in a range of a few hundred MeV. The energy of the beam was scanned over 250 $\mathrm{MeV}$ in $50 \mathrm{MeV}$ steps. This scan was clearly tracked by the spectrometer (Fig. 5), although the step size was found to be different from the feedback setting. In addition, spectrometer data were compared to the data from the BPMs located at the high dispersion points in the ESA extraction line and a good correlation was observed.

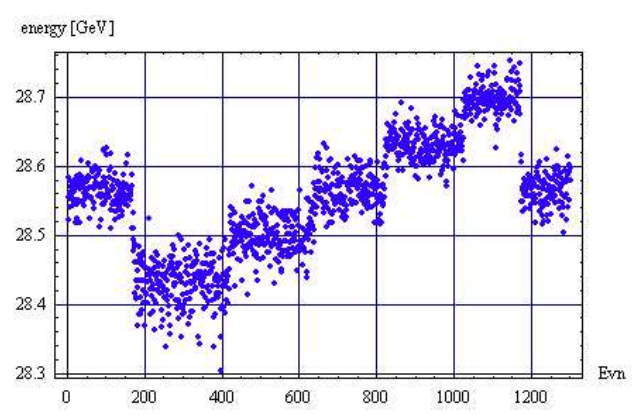

Figure 5: Energy scan as seen by the spectrometer.

\section{SUMMARY}

We had four data taking periods at SLAC's End Station A in 2006-2007. The performance of the BPM system was tested and the first data providing energy measurement from a full model of the proposed spectrometer were obtained. We demonstrated sub-micron precision of the orbit reconstruction using cavity BPMs, systematic drifts of about $1 \mu \mathrm{m}$ over one hour of operation were observed. Our futher studies will focus on long term stability issues of the hardware and address the absolute accuracy of the beam position and energy measurements.

\section{REFERENCES}

[1] R. Assmann et al, Calibration of Center-of-Mass Energies at LEP2 for a precise measurement of the W Boson Mass, CERN-PH-EP-2004-032, 2004

[2] D. Whittum, Y. Kolomensky, Analysis of an Asymmetric Resonant Cavity as a Beam Monitor, Review of Scientific Instruments, V. 70, N 5, May 1999

[3] R. Arnold et al., Magnetic Measurements and Simulations of a 4-Magnet Chicane..., These proceedings 